Reprod. Nutr. Dévelop., 1984, 24 (4), 361-378.

\title{
Démonstration génétique de l'inversion fonctionnelle du phénotype sexuel femelle sous l'action de la température d'élevage chez l'Amphibien Urodèle: Pleurodeles waltlii Michah
}

\author{
C. DOURNON, Ch. HOUILLON
}

avec la collaboration technique de Madame SZMUTYK, de Messieurs DESROSIERS et LORTHIOIT

Laboratoire de Biologie animale, Université $P$. et $M$. Curie, 9 quai Saint-Bernard, 75230 Paris cedex 05, France

Summary. Genetic demonstration of functional sex inversion in Pleurodeles waltlii Michah. (urodele amphibian) under the effect of temperature.

Rearing standard Pleurodeles waltlii at ambient temperature always leads to a female to male sex ratio of $1: 1$ (639 $\&-617 \sigma^{\prime \prime} ; 20$ matings). Larvae known to be of ZW female sex genotype were reared at $30^{\circ} \mathrm{C}$ for 4 months (from stage 33 to 2 months after metamorphosis). When they were adult, the majority (11 out of 15) displayed all the characters of male sexual maturity (heat-induced $Z W$ neo-males). These neo-males were functional. The offspring were analysed at ambient temperature. When heat-induced $Z W$ neo-males were bred with ZW standard females, the sex of the offspring conformed to the theoretical expectation of 3 females to 1 male $\left(691 q-247 \sigma^{x} ; 14\right.$ matings). Some females from the preceding matings displayed the new WW genotype. When they were mated with $Z Z$ standard males, the offspring were $100 \%$ phenotypic females (587 $\%$; matings). Breeding heat-induced $Z W$ neo-males with WW females gave $100 \%$ phenotypic females (201 \& ; 2 matings).

These results provide the first irrefutable genetic demonstration of temperature sexreversal in tetrapode vertebrates.

\section{Introduction.}

L'action de la température d'élevage sur le développement des Amphibiens a été signalée pour la première fois par Hertwig (1898) : une élévation de la température réduit la durée du développement de Rana fusca (= Rana temporaria) et de Rana esculenta. Witschi $(1914,1929)$ a constaté qu'une température anormalement élevée modifiait la différenciation des gonades de Rana temporaria et de Rana sy/vatica. A sa suite, d'autres auteurs se penchèrent sur le problème surtout chez les Anoures : Rana temporaria et Bufo vulgaris (Piquet, 1930), Rana japonica (Yoshikura, 1959, 1963), Rana catesbeiana (Hsü, Yü et Liang, 1971) et seulement sur une espèce d'Urodèle: Hynobius retardatus (Uchida, 1937). D'une manière générale, les auteurs s'accordent pour admettre que les larves d'Amphibiens élevées à la température de 25 à $32{ }^{\circ} \mathrm{C}$ évoluent selon le phénotype mâle et celles 
élevées à basse température $\left(10^{\circ} \mathrm{C}\right)$ selon le phénotype femelle. L'inversion du phénotype sexuel par une température d'élevage très différente de la normale n'était qu'une simple déduction basée sur l'obtention de sex-ratio déviées, établies à partir d'individus en cours de métamorphose ou juvéniles. Aucun de ces animaux n'avait été élevé jusqu'à l'état adulte en vue de la reproduction ; leur race sexuelle (indifférenciée, semi-différenciée ou différenciée) n'était pas toujours clairement définie et leur génotype sexuel n'était jamais connu. De la sorte, aucune preuve de l'inversion fonctionnelle d'un phénotype sexuel sous l'action de la température $n^{\prime}$ avait encore été apportée.

Le problème méritait d'être repris chez Pleurodeles walt/ii, Urodèle de race sexuelle différenciée. Cet Amphibien offre l'avantage d'avoir largement été utilisé pour l'inversion du sexe par diverses techniques. L'homogamétie mâle $Z Z$ et I'hétérogamétie femelle $\mathrm{ZW}$ ont été clairement démontrées. Le traitement au benzoate d'œstradiol appliqué pendant la vie larvaire a permis à Gallien (1950) d'obtenir des néo-femelles $Z Z$ qui croisées avec des mâles standard $Z Z$, ont donné des descendances monogéniques mâles ZZ (Gallien, 1951). Par des greffes embryonnaires du type de celles qu'Humphrey (1928, 1931, 1945) réalisa chez l'Axolotl, Collenot (1973) a pu, chez Pleurodeles waltlii, obtenir des néo-mâles ZW qui croisés avec des femelles normales ZW ont donné parmi leurs descendants des femelles thélygènes WW. Ces dernières croisées avec des mâles standard donnent des descendances monogéniques femelles $\mathrm{ZW}$.

L'élevage à la température de $30^{\circ} \mathrm{C}$ d'embryons à génotype femelle connu est à l'origine du présent travail qui apporte pour la première fois chez les Vertébrés Tétrapodes les preuves irréfutables de l'inversion fonctionnelle du sexe liée à l'action de la température.

\section{Matériel et méthodes.}

Conditions d'élevage. - Les conditions d'élevage et de reproduction de Pleurodeles waltlii en laboratoire ont été précisées par Gallien (1952). Les conditions considérées comme normales dans ce travail correspondent à l'élevage pratiqué depuis la ponte jusqu'à l'état adulte à la température ambiante du laboratoire $\left(20 \pm 2^{\circ} \mathrm{C}\right)$. Les conditions expérimentales consistent à élever les embryons depuis la ponte jusqu'au stade 33 (éclosion) à savoir une semaine à la température ambiante, puis à pratiquer l'élevage à la température de $30^{\circ} \mathrm{C}$ pendant 4 mois pour revenir ensuite aux conditions normales. Les animaux supportent bien un tel traitement thermique pendant une aussi longue période ; la mortalité n'est guère plus importante que dans les conditions normales. II convient cependant de veiller à la propreté de l'eau qui se pollue rapidement à $30^{\circ} \mathrm{C}$. De plus, une plus grande attention doit être portée aux animaux afin d'éviter les noyades fréquentes à l'époque de la métamorphose. Celle-ci intervient 2 mois après le début du traitement thermique et se produit environ 1 mois plus tôt qu'à la température ambiante.

Les stades morphologiques sont ceux définis dans la table du développement de Gallien et Durocher (1957).

Recherche du phénotype sexuel. - Dans les conditions normales d'élevage le sexe de la gonade est reconnaissable histologiquement à partir du stade 53 
(larve âgée de 2 mois). Peu avant la métamorphose, c'est-à-dire environ 1 mois plus tard, on peut reconnaître un testicule ou un ovaire d'après leur aspect morphologique au moment de la dissection. Mais quand il est nécessaire de garder les animaux jusqu'à l'état adulte, la reconnaissance du sexe de la gonade doit se faire au cours d'une laparotomie. Celle-ci est praticable vers l'âge de 5 mois pour les Pleurodèles élevés à $20 \pm 2{ }^{\circ} \mathrm{C}$. L'intervention chirurgicale consiste, après anesthésie, à inciser le flanc de l'animal afin d'observer la morphologie de ses glandes génitales, puis à poser quelques points de suture avant de remettre l'animal dans son milieu aquatique (fig. 2).

Recherche du génotype sexuel. - Chez Pleurodeles waltlii, il n'existe aucun dimorphisme des chromosomes mitotiques qui permette de reconnaître la paire de chromosomes sexuels. Pour cet Urodèle, on ne dispose actuellement que de deux méthodes pour préciser le génotype sexuel : l'analyse de la descendance et la détection d'une enzyme liée au sexe (Ferrier et al., 1980, 1983). Nous avons utilisé l'analyse de la descendance qui est la base de nos premiers résultats (Houillon et Dournon, 1978) et qui est particulièrement fiable en raison de la très faible dispersion statistique des nombres de mâles et de femelles autour des distributions théoriques. La méthode basée sur la détection de la peptidase-1 d'origine érythrocytaire comme marqueur du sexe génétique fut utilisée dès sa mise au point. Nous avons alors constaté que dans certaines souches de Pleurodèles de notre laboratoire, un individu sur deux à génotype $\mathrm{ZW}$ connu présentait le même spectre enzymatique que les mâles standard $Z Z$. Il est évident qu'en présence de tels animaux, la méthode enzymatique ne peut être appliquée à la détection du génotype sexuel. Cette méthode garde toute sa valeur dans la mesure où les phénotypes enzymatiques parentaux sont connus, ce qui n'était pas le cas lors de nos premières séries expérimentales.

Pour l'analyse de la sex-ratio des descendances, les nombres de mâles et de femelles sont comparés par le test de conformité du $\varkappa^{2}$ aux nombres théoriques de mâles et de femelles déterminés par les combinaisons possibles entre le génotype connu d'un Pleurodèle et les trois possibilités génotypiques $Z Z, Z W$ et WW de l'individu testé (tabl. 1). Pour l'analyse des résultats, nous avons choisi un seuil de signification défini pour un risque de 0,05 et pour 1 degré de liberté. Ce seuil est de 3,841 selon la table du $\varkappa^{2}$.

Les études statistiques requièrent un grand nombre d'animaux, d'où la

TABLEAU 1

Pourcentages théoriques de mâles et de femelles phénotypiques issus de croisements entre des individus témoins ( $\sigma^{*} \mathrm{ZZ}$ et $९ \mathrm{ZW}$ standard) et des individus expérimentaux.

\begin{tabular}{|c|c|c|c|}
\hline \multicolumn{2}{|r|}{ Parents } & \multicolumn{2}{|c|}{$\begin{array}{l}\text { Sex-ratio théorique } \\
\text { de la descendance } \\
\% \text { o }\end{array}$} \\
\hline $\mathrm{O}^{*} \mathrm{ZZ}$ (standard) & $\left\{\begin{array}{lll}9 & Z Z & \text { (néo-femelle) } \\
9 & \mathrm{ZW} & \text { (standard) } \\
\wp & W W & \text { (thélygène) }\end{array}\right.$ & $\begin{array}{r}100 \\
50 \\
0\end{array}$ & $\begin{array}{r}0 \\
50 \\
100\end{array}$ \\
\hline @ ZW (standard) $>$ & $\left\{\begin{array}{lll}\text { o } & \text { ZZ } & \text { (standard) } \\
\text { o* ZW } & \text { (néo-mâle) } \\
0^{\circ} \text { WW } & \text { (néo-mâle) }\end{array}\right.$ & $\begin{array}{r}50 \\
25 \\
0\end{array}$ & $\begin{array}{r}50 \\
75 \\
100\end{array}$ \\
\hline
\end{tabular}


nécessité d'importants élevages. Rappelons que le Pleurodèle atteint la maturité sexuelle dans les conditions normales vers l'âge de 12-14 mois pour le mâle et de 15-16 mois pour la femelle. La sex-ratio de la descendance ne peut être établie d'une façon commode qu'avec des animaux juvéniles âgés d'au moins 5 mois. Ainsi, à partir de jeunes larves dont l'inversion est tentée, l'identification du génotype sexuel et par là même la preuve de l'inversion, ne peut être obtenue qu'environ 2 années plus tard en tenant compte du repos sexuel pendant la période estivale.

La démarche expérimentale qui a été suivie est représentée sur la figure 1.

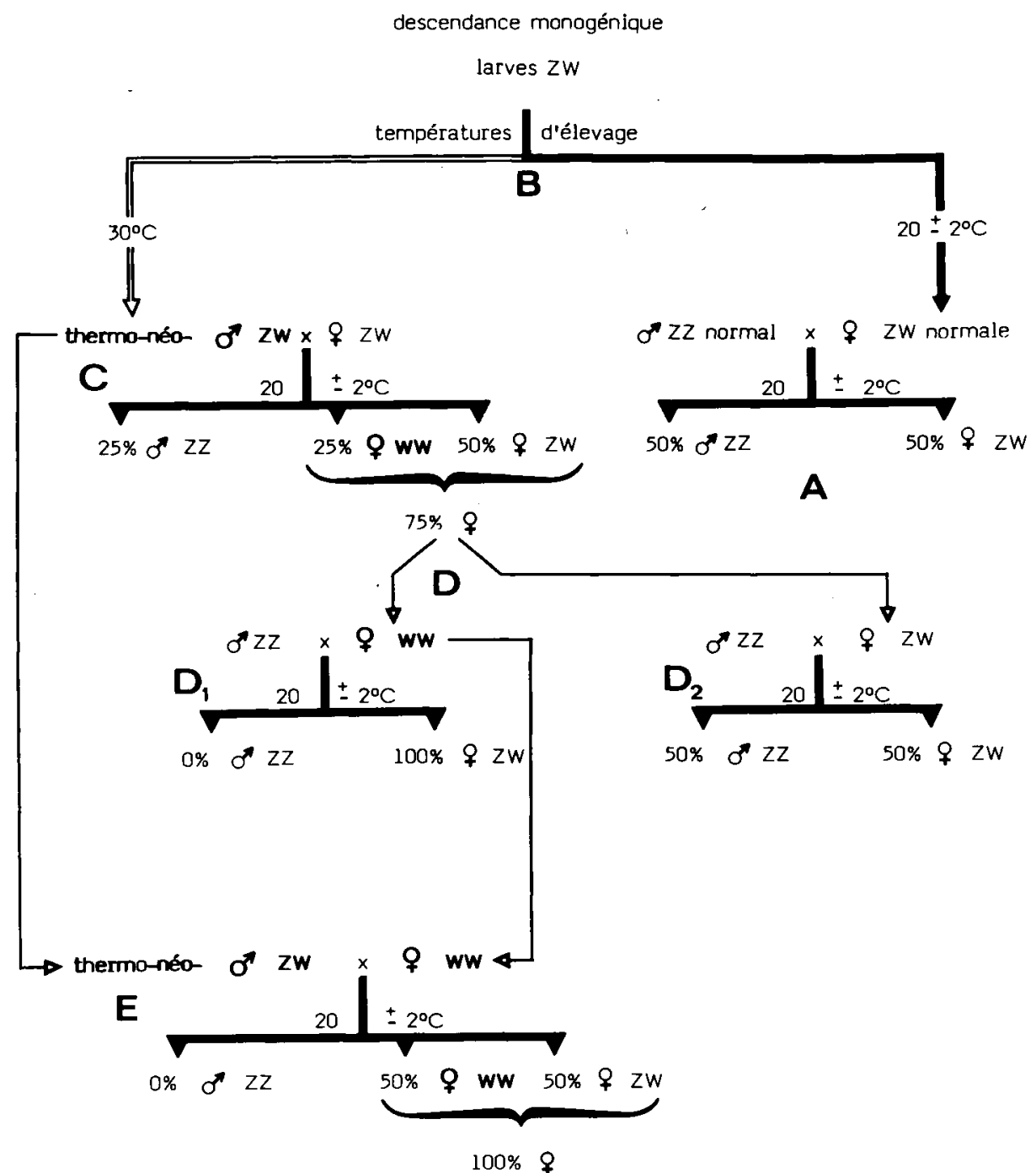

FIG. 1. - Démarche expérimentale suivie pour la démonstration génétique de l'inversion fonctionnelle du phénotype sexuel femelle sous l'influence de la température chez Pleurodeles waltiii. Les pourcentages indiqués sont les pourcentages théoriques attendus dans les divers croisements. 


\section{Résultats et interprétation.}

1. Sex-ratio des Pleurodèles élevés à la température du laboratoire. La sex-ratio de la descendance issue du croisement entre un mâle $Z Z$ et une femelle $Z W$ est théoriquement de $50 \%$ de mâles et $50 \%$ de femelles (fig. 1A). Il est évident que ce résultat n'a qu'une valeur statistique et qu'il peut présenter des variations non seulement selon les pontes mais aussi, d'après nos observations, selon les conditions d'élevage. II est donc nécessaire de connaître d'abord l'amplitude des variations de la sex-ratio dans les conditions normales d'élevage à $20 \pm 2{ }^{\circ} \mathrm{C}$ avant de pouvoir donner une signification à la sex-ratio après l'élevage à $30^{\circ} \mathrm{C}$.

Les résultats présentés dans le tableau 2 se rapportent aux descendances issues de 20 croisements différents, élevées séparément, et à un regroupement d'animaux issus de divers croisements pour lesquels seulement un nombre réduit d'individus était obtenu. Sur un total de 1256 Pleurodèles élevés dans les conditions de température ambiante $\left(20 \pm 2{ }^{\circ} \mathrm{C}\right)$, les mâles constituent $49,1 \%$ de la population et les femelles 50,9\%. II n'a jamais été observé de Pleurodèle intersexué dans les élevages à la température du laboratoire. Dans tous les cas, le test de conformité du $\varkappa^{2}$ n'est pas significatif ; les résultats sont donc conformes à la prévision théorique.

D'après les résultats de Gallien (1954) et de Collenot (1973) et d'après nos propres résultats, l'élevage de Pleurodeles waltlii dans les conditions habituelles

TABLEAU 2

Etude de la descendance de Pleurodèles standard, élevés à la température du laboratoire $\left(20 \pm 2^{\circ} \mathrm{C}\right)$.

\begin{tabular}{|c|c|c|c|c|}
\hline $\begin{array}{l}\text { Référence de la } \\
\text { descendance }\end{array}$ & $\begin{array}{c}\text { Nombre de } \\
\sigma^{*}\end{array}$ & $\begin{array}{c}\text { Nombre de } \\
\wp\end{array}$ & Total & $\begin{array}{c}\chi^{2} \\
\text { (seuil : } 3,841 \text { ) }\end{array}$ \\
\hline Sd $76 a$ & 17 & 13 & 30 & 0,533 \\
\hline Sd $76 b$ & 20 & 17 & 37 & 0,243 \\
\hline A $\quad 4-77$ & 29 & 37 & 66 & 0,970 \\
\hline $19-77$ & 20 & 20 & 40 & 0 \\
\hline $24-77$ & 37 & 37 & 74 & 0 \\
\hline $4-77$ & 10 & 11 & 21 & 0,048 \\
\hline $3-77$ & 30 & 29 & 59 & 0,017 \\
\hline $4-77$ & 25 & 25 & 50 & 0 \\
\hline $1-77$ & 48 & 42 & 90 & 0,400 \\
\hline $2-77$ & 26 & 36 & 62 & 1,613 \\
\hline 3-77 & 28 & 32 & 60 & 0,267 \\
\hline $4-77$ & 24 & 28 & 52 & 0,308 \\
\hline $5-77$ & 19 & 14 & 33 & 0,757 \\
\hline $7-77$ & 14 & 17 & 31 & 0,290 \\
\hline Sd 77 & 43 & 49 & 92 & 0,391 \\
\hline Sd $78 a$ & 24 & 33 & 57 & 1,421 \\
\hline $\mathrm{Sd} 78 \mathrm{~b}$ & 35 & 32 & 67 & 0,134 \\
\hline Sd $1-79 a$ & 13 & 15 & 28 & 0,143 \\
\hline Sd $1-79 b$ & 23 & 25 & 48 & 0,083 \\
\hline Sd $2-79$ & 39 & 46 & 85 & 0,576 \\
\hline Pontes regroupées & 93 & 81 & 174 & 0,828 \\
\hline Total & 617 & 639 & 1256 & \\
\hline
\end{tabular}


de température du laboratoire conduit à une sex-ratio conforme à la prévision théorique. Il est logique de considérer que dans les conditions où la température oscille de quelques degrés autour de $20^{\circ} \mathrm{C}$ pendant le développement larvaire, le phénotype sexuel du Pleurodèle s'exprime conformément au génotype sexuel.

\section{Inversion du phénotype sexuel femelle sous l'action d'une température de $30^{\circ} \mathrm{C}$.}

2.1. Mise en évidence. - La démonstration simple et sans ambiguïté de l'inversion du phénotype sexuel femelle est basée sur l'utilisation d'une descendance monogénique femelle $Z W$. Si par un artifice d'élevage, on peut obtenir ne serait-ce qu'un très petit nombre de mâles phénotypiques, ou même un seul, on pourra affirmer qu'il s'agit de néo-mâles ZW, c'est-à-dire de mâles phénotypiques mais de génotype sexuel femelle.

Grâce à l'obligeance du Professeur Collenot, nous avons pu disposer de 60 embryons de Pleurodeles waltlii provenant d'une descendance monogénique femelle ZW. Ces embryons à génotype sexuel connu ont d'abord été élevés à la température ambiante du laboratoire. A partir du stade 33 (éclosion), les jeunes larves ont été réparties en deux lots (fig. 1, B) :

- un lot témoin constitué de 20 larves dont l'élevage s'est poursuivi à la température ambiante $\left(20 \pm 2{ }^{\circ} \mathrm{C}\right)$,

- un lot expérimental comprenant 40 larves élevées à la température de $30^{\circ} \mathrm{C}$ pendant 4 mois.

Pour le lot témoin, il restait 10 animaux à l'âge de 5 mois. La laparotomie a confirmé qu'il s'agissait bien de femelles. La maturité sexuelle fut atteinte vers l'âge de 16 mois. Croisées avec des mâles témoins, ces femelles ont donné une descendance à sex-ratio normale qui confirme leur génotype $\mathrm{ZW}$.

Les animaux du lot expérimental élevés à la température de $30^{\circ} \mathrm{C}$ dès le stade de l'éclosion se sont métamorphosés trois semaines plus tôt que les témoins. Ils mesuraient seulement 55 à $60 \mathrm{~mm}$ alors qu'à la métamorphose les Pleurodèles

FIG. 2. - Tractus uro-génital juvénile d'un mâle et d'une femelle standard au moment de la laparotomie (5 mois, $80 \mathrm{~mm}$ ).

FIG. 3. - Coupe longitudinale d'un testicule de thermo-néo-male ZW.

La structure est comparable à celle d'un mâle standard.

FIG. 4. - Tractus uro-génital d'un mâle standard adulte (15 mois, $130 \mathrm{~mm}$ ).

FIG. 5. - Tractus uro-génital d'une femelle standard adulte (15 mois, $140 \mathrm{~mm})$.

FIG. 6. - Tractus uro-génital d'un intersexué ZW (20 mois, $115 \mathrm{~mm})$.

FIG. 7. - Tractus uro-génital d'un thermo-néo-mâle ZW adulte (15 mois, $120 \mathrm{~mm}$ ). Même aspect que celui d'un mâle standard.

cy : cystes ; ca : callosités ; $\mathrm{cl}$ : cloaque ; $\mathrm{CM}$ : canal de Müller ; $\mathrm{CW}$ : canal de Wolff ; o : ovaire et ovocytes ; s : spermatozoïdes ; $t$ : testicule. 


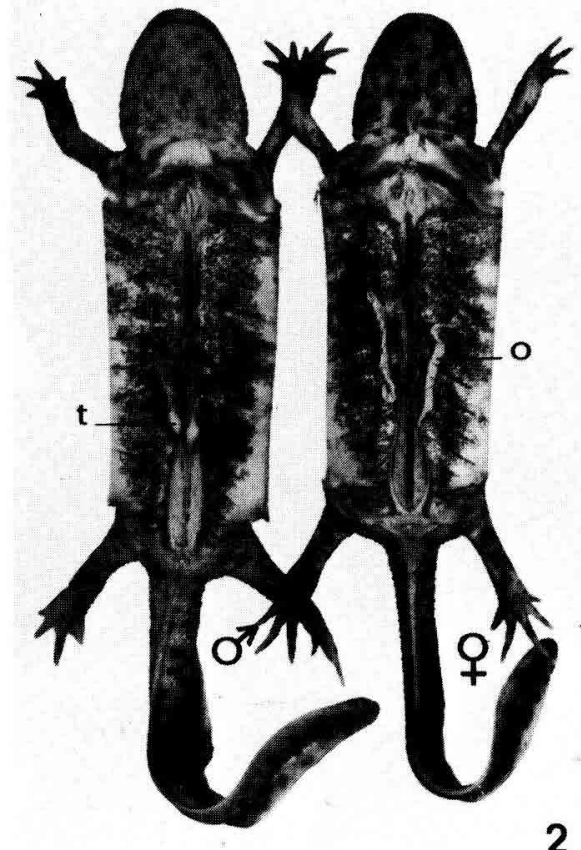

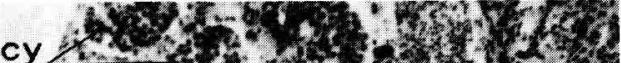
(6.5.

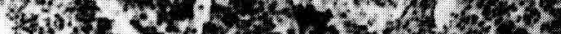

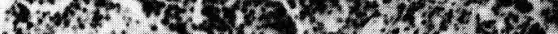

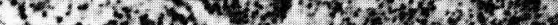

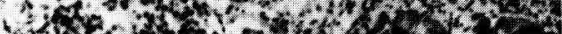

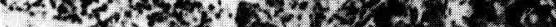

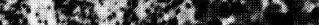

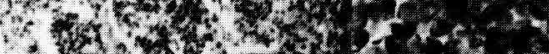

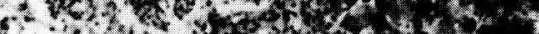

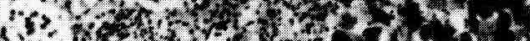

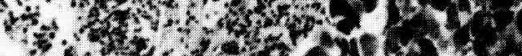

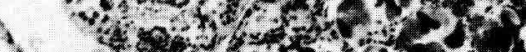

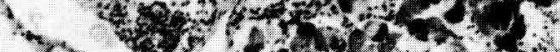

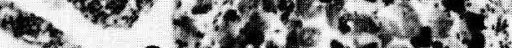

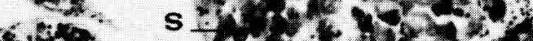

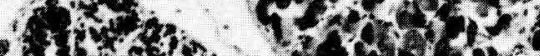

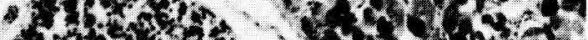

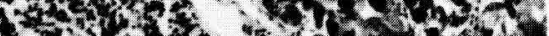

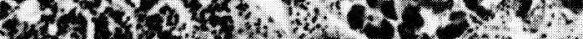

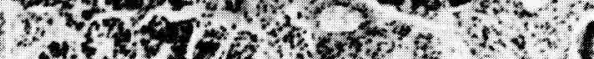

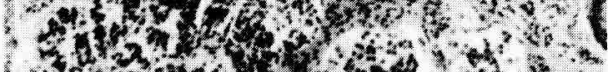

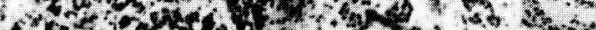

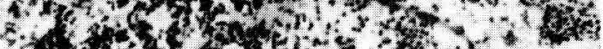

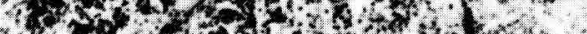

2 ghe

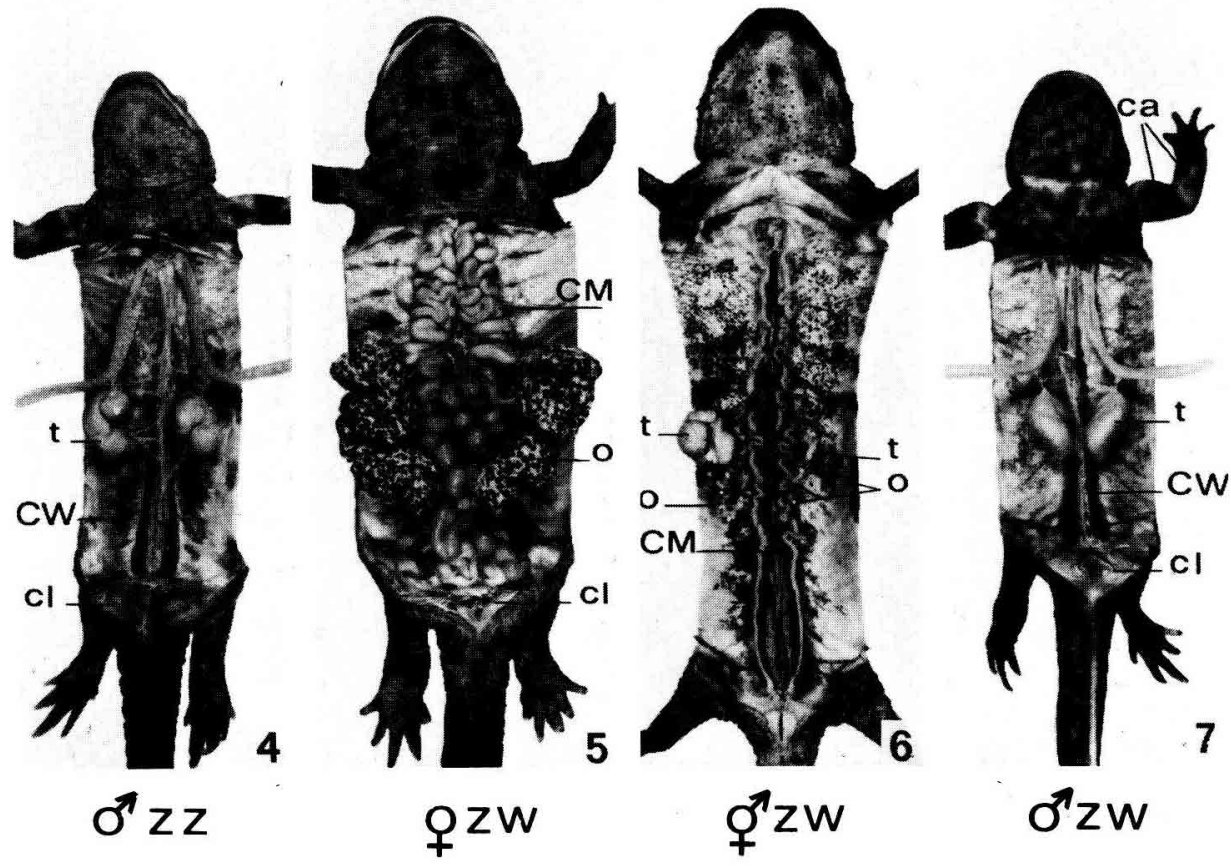


mesurent en moyenne $70 \mathrm{~mm}$. Le traitement thermique s'est poursuivi encore 2 mois après la métamorphose. A la fin du traitement, il restait 15 animaux âgés de 18 semaines qui furent replacés dans les conditions normales d'élevage.

Dès cet âge, 4 Pleurodèles présentaient une légère turgescence du cloaque et quelques semaines plus tard les callosités apparaissaient sur les membres antérieurs. A l'âge de 6 mois, certains jeunes mâles étaient déjà capables de s'accoupler. A 7 mois, 10 animaux avaient des callosités, et à 12 mois, 11 Pleurodèles sur 15 présentaient tous les caractères externes de la maturité sexuelle mâle. La laparotomie des 4 autres animaux de ce lot a révélé qu'il s'agissait de femelles ayant un tractus uro-génital tout à fait normal (fig. 5). Deux d'entre elles se sont reproduites à l'âge de deux ans avec des mâles standard ZZ. La sex-ratio de leur descendance a confirmé leur génotype $Z W$. En ce qui concerne le tractus uro-génital des 11 Pleurodèles de phénotype mâle, la laparotomie ou la dissection a révélé un tractus mâle tout à fait normal pour 9 d'entre eux (fig. 7). L'observation histologique de leurs testicules révèle la présence de nombreux spermatozoïdes et une structure générale identique à celle des testicules témoins à maturité sexuelle (fig. 3). Les 2 autres individus, bien qu'ayant présenté tous les caractères externes de la maturité sexuelle mâle (cloaque turgescent et callosités aux membres antérieurs) avaient des gonades intersexuées (fig. 6).

Ces observations apportent les deux premières preuves de l'inversion du phénotype sexuel femelle sous l'action de la température d'élevage de $30^{\circ} \mathrm{C}$. Ces preuves sont :

- des animaux ayant un génotype femelle ZW connu ont évolué en mâles phénotypiques. Le terme de "thermo-néo-mâle " a été donné à ces animaux pour souligner qu'ils sont obtenus par un traitement thermique et que leur phénotype sexuel ne correspond pas à leur génotype sexuel ;

- des animaux ZW sont intersexués ce qui montre que l'inversion phénotypique peut n'être que partielle.

\subsection{Analyse de la descendance des thermo-néo-mâles ZW.}

2.2.1. Sex-ratio. - Les 9 thermo-néo-mâles et les 2 intersexués précédents ainsi que 12 autres thermo-néo-mâles provenant d'expériences ultérieures analogues à celle présentée ci-dessus ont été accouplés avec des femelles vierges ZW (fig. 1, C). A partir de ces croisements, 14 pontes fécondées ont été obtenues et élevées séparément à la température ambiante du laboratoire. A l'âge de 5 mois, soit un mois environ après la métamorphose, les animaux ont été sacrifiés ou ont subi une laparotomie afin de connaître le sexe de leurs gonades.

Les résultats du tableau 3 font nettement apparaître un nombre réduit de mâles par rapport au nombre important de femelles. C'est ainsi que sur un total de 1192 Pleurodèles issus de 14 croisements différents, il y a 341 mâles $(28,6 \%)$ et 851 femelles $(71,4 \%)$. D'après la prévision théorique, la descendance issue du croisement entre un thermo-néo-mâle $Z W$ et une femelle standard $Z W$ doit comprendre $25 \%$ de mâles à génotype $Z Z, 50 \%$ de femelles à génotype $Z W$ et $25 \%$ d'animaux à génotype particulier WW. Or les 14 descendances analysées ayant donné $28,6 \%$ de mâles et $71,4 \%$ de femelles, il faut admettre que la constitution 
TABLEAU 3

Descendances issues des croisements entre thermo-néo-mâles $Z W$ et femelles standard $Z W$ vierges lélevage à $20 \pm 2{ }^{\circ} \mathrm{C}$ ).

\begin{tabular}{|c|c|c|c|c|c|}
\hline \multirow{2}{*}{$\begin{array}{c}\text { Référence } \\
\text { de la } \\
\text { descendance }\end{array}$} & \multirow[b]{2}{*}{$\begin{array}{c}\text { Nombre de } \\
\sigma^{\circ}\end{array}$} & \multirow[b]{2}{*}{$\begin{array}{c}\text { Nombre de } \\
\&\end{array}$} & \multirow[b]{2}{*}{ Total } & \multicolumn{2}{|c|}{ Prévision théorique } \\
\hline & & & & $1 \circ-3$ q & $1 \% \bar{x}^{2}+9$ \\
\hline A-77 & 8 & 33 & 41 & 0,658 & 15,244 \\
\hline B-77 & 40 & 144 & 184 & 1,043 & 58,783 \\
\hline C-77 & 21 & 58 & 79 & 0,105 & 17,329 \\
\hline D-77 & 7 & 26 & 33 & 0,253 & 10,939 \\
\hline$E-77$ & 8 & 17 & 25 & 0,653 & 3,240 \\
\hline F-77 & 28 & 76 & 104 & 0,205 & 22,154 \\
\hline G-77 & 18 & 40 & 58 & 1,126 & 8,345 \\
\hline $\mathrm{H}-79$ & 38. & 88 & 126 & 1,788 & 19,841 \\
\hline I-81 & 8 & 19 & 27 & 0,309 & 4,481 \\
\hline $\mathrm{J}-81$ & 50 & 127 & 177 & 0,996 & 33,497 \\
\hline (*) $^{*}$ K-81 & 45 & 81 & 126 & 7,714 & 10,286 \\
\hline (*) $^{*} \quad$ L-82 & 49 & 79 & 128 & 12,042 & 7,031 \\
\hline$M-82$ & 4 & 13 & 17 & 0,019 & 4,765 \\
\hline N-82 & 17 & 50 & 67 & 0,005 & 16,254 \\
\hline \multirow{2}{*}{$\left.\begin{array}{l}\text { Total } \\
\text { Total sans } \\
\text { K-81 et } L-82\end{array}\right\}$} & 341 & 851 & 1192 & & \\
\hline & 247 & 691 & 938 & & \\
\hline
\end{tabular}

${ }^{(*)}$ Sex-ratio s'écartant de la prévision théorique (interprétation dans le texte).

génotypique sexuelle WW est viable et qu'elle correspond à un phénotype femelle. Ceci confirme les résultats antérieurs obtenus à partir de greffes embryonnaires (Collenot, 1973).

II est essentiel de noter que dans ces descendances le génotype sexuel WW doit théoriquement concerner un tiers des femelles.

Exception faite des descendances $\mathrm{K}-81$ et $\mathrm{L}-82$, pour chaque descendance considérée individuellement, le test de conformité indique qu'il n'y a aucune différence significative entre les nombres observés de mâles et de femelles et les nombres théoriques de mâles et de femelles. La sex-ratio obtenue $(26,3 \%$ de mâles et $73,7 \%$ de femelles) est conforme aux prévisions théoriques. Les mâles parentaux ont bien le génotype sexuel $Z W$. En outre, le test de conformité montre de façon significative qu'il ne s'agit pas de mâles $Z Z$ lesquels auraient donné une descendance composée de $50 \%$ d'individus de l'un et l'autre sexe.

Les descendances $\mathrm{K}-81$ et $\mathrm{L}-82$ ont une sex-ratio particulière, voisine de 1 mâle pour 2 femelles. L'analyse montre que cette sex-ratio présente une différence statistiquement significative par rapport à la descendance de mâles standard ZZ (1 mâle pour 1 femelle) d'une part, mais aussi de thermo-néo-mâles ZW (1 mâle pour 3 femelles) d'autre part. Par contre, la conformité est bonne avec une proportion théorique de $33,3 \%$ de mâles et de $66,7 \%$ de femelles ; $\varkappa^{2}$ est égal à 0,321 pour la descendance $K-81$ et à 1,395 pour L-82, soit bien inférieur au seuil 3,841 , ce qui n'est pas le cas des 12 autres descendances.

La sex-ratio particulière des descendances K-81 et L-82 dépendrait d'une létalité en rapport avec la consanguinité des animaux WW et la peptidase-1. Ferrier et 
al. (1983), Cayrol et al. (1983) ont montré que cette enzyme est dimérique, polymorphe et dépend d'un couple d'allèles codominants localisés sur le chromosome sexuel $Z\left(P\right.$ ep- $\left.1^{A}\right)$ et sur le chromosome sexuel W (Pep-1B). Cependant dans la lignée de Pleurodèle 103, l'allèle situé sur le chromosome W serait « nul » ou " silencieux $"\left(P\right.$ ep- $\left.1^{0}\right)$. Les femelles standard ZW sont normalement Pep- $1^{A} /$ Pep- $1^{B}$ et leur zymogramme comporte trois bandes; certaines femelles ZW sont Pep$1^{A} /$ Pep- $1^{0}$, leur zymogramme ne comporte qu'une seule bande lente.

Dans nos élevages, un Pleurodèle sur deux à génotype ZW est Pep- $1 \mathrm{~A} / \mathrm{Pep}-1^{0}$. Le croisement entre deux individus de ce type doit conduire à des descendants WW qui seront Pep-1\% Pep- $1^{\circ}$. Cayrol et al. (1983) ont effectivement reconnu de tels animaux mais il s'agissait de Pleurodèles obtenus par gynogenèse. A la suite de nos croisements, il n'a été reconnu aucun animal Pep- $1 \% /$ Pep- $1^{\circ}$. Il y a une corrélation entre l'absence de ces animaux et la sex-ratio ( 1 mâle pour 2 femelles) des descendances $\mathrm{K}-81$ et L-82 ; l'interprétation résiderait dans la létalité des animaux ayant la constitution chromosomique $W W$, particulière à ces deux descendances.

Quoi qu'il en soit, les descendances de 12 thermo-néo-mâles ZW conformes à la prévision théorique prouvent d'une façon évidente que ces animaux sont fertiles.

Une telle analyse génétique est fort longue, cependant la maturité sexuelle précoce manifestée dès l'âge de 5 mois par certains thermo-néo-mâles, a permis de réduire la durée des protocoles expérimentaux. Ainsi un thermo-néo-mâle ZW âgé de 5 mois et 3 semaines et mesurant $60 \mathrm{~mm}$ a été croisé avec une femelle standard âgée de 14 mois et mesurant $180 \mathrm{~mm}$. De la sorte, il a été possible d'obtenir au cours de la même année le cycle complet d'un Pleurodèle mâle et de disposer de sa descendance sexable par laparotomie vers l'âge de 5 mois, alors que dans les conditions habituelles le protocole requiert au moins 2 années.

II convient de remarquer que c'est la première fois qu'est obtenue chez Pleurodeles waltlii, la reproduction d'un mâle à peine âgé de 6 mois et de taille aussi réduite alors que dans les conditions normales, il est assez exceptionnel d'obtenir la reproduction des mâles dès l'âge de 10 mois $(120 \mathrm{~mm}$ de longueur).

2.2.2. Détection des femelles thélygènes WW. - Aucune indication morphologique, anatomique ou caryologique ne permet de discerner une femelle à génotype WW d'une femelle à génotype normal ZW. Le génotype WW ne peut être détecté qu'à la faveur de nouveaux croisements entre des mâles standard $Z Z$ et les femelles issues de la descendance des thermo-néo-mâles ZW (fig. 1, D). En vue de cette détection, les femelles issues de la descendance de 3 thermo-néo-mâles différents (descendances A-77, B-77 et E-77 du tableau 3) ont été élevées jusqu'à l'état adulte. Au moment de la laparotomie à 5 mois, les 3 descendances comprenaient au total 250 animaux dont 56 mâles $(22,4 \%)$ et 194 femelles $(77,6 \%)$. Parmi ces dernières, 32 ont été croisées avec des mâles standard et se sont reproduites ; leurs progénitures ont ensuite été élevées dans les conditions de température ambiante. 27 femelles parmi les 32 ont donné une descendance bisexuée comprenant au total 389 mâles $(48,9 \%)$ et 408 femelles $(51,1 \%)$. Par contre, les 5 autres femelles ont donné une descendance unisexuée comprenant respectivement : 54 , $70,81,112$ et 270 femelles soit au total 587 femelles (100\%) (tabl. 4). 


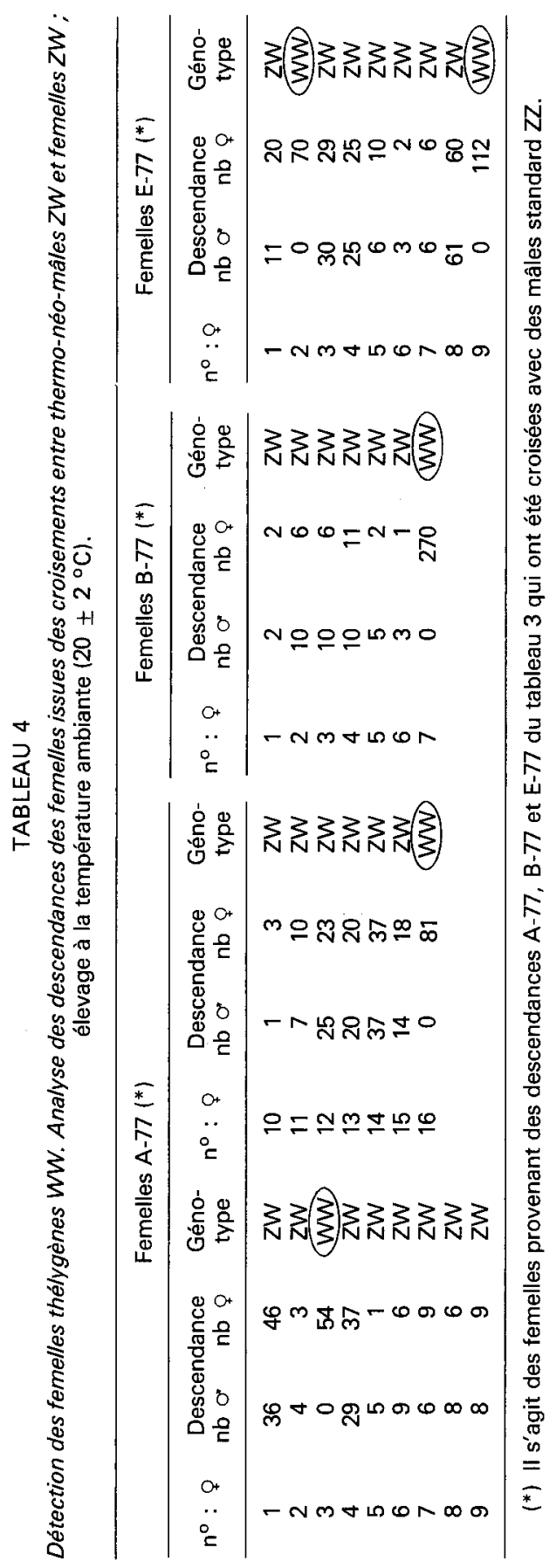


Selon la prévision théorique, une femelle ayant une descendance bisexuée possède le génotype sexuel $\mathrm{ZW}$, tandis qu'une femelle ayant une descendance unisexuée femelle possède le génotype sexuel WW. Dès lors, l'importance de la descendance unisexuée obtenue pour les 5 femelles permet d'affirmer qu'elles possèdent le génotype sexuel WW (femelles thélygènes). La population de femelles parmi laquelle étaient recherchées les femelles thélygènes comprend théoriquement $2 / 3$ de femelles $Z W$ et $1 / 3$ de femelles $W W$. Sur les 32 femelles qui ont pu se reproduire, on pouvait s'attendre à détecter une dizaine de femelles thélygènes. Or seulement 5 ont donné une descendance unisexuée femelle. Il a été indiqué précédemment l'éventualité d'une moins grande viabilité des Pleurodèles en relation avec la constitution chromosomique WW dans certaines descendances. Ceci n'est pas le cas pour les femelles de la population testée puisqu'elles proviennent de 3 descendances qui comportaient à l'âge de 5 mois $22,4 \%$ de mâles et $77,6 \%$ de femelles. La population testée semblait présenter le maximum de garanties pour la détection des femelles WW. Mais si les pourcentages étaient particulièrement probants pour des animaux âgés de 5 mois, il n'en est peut-être plus de même 2 ans plus tard lorsque les femelles sont en âge de se reproduire. Une fragilité des animaux en relation avec la constitution WW a pu entre temps entraîner la disparition préférentielle des femelles WW. Cette interprétation est difficile à vérifier compte tenu des servitudes imposées par l'élevage d'un grand nombre d'animaux adultes. Mais d'autres interprétations peuvent expliquer ce nombre réduit de femelles WW, par exemple une fécondité plus faible que celle des femelles standard ZW, d'où les aléas de la détection du génotype WW.

Néanmoins, la présence des femelles WW confirme à son tour le génotype ZW des thermo-néo-mâles parentaux et constitue une nouvelle preuve de l'inversion fonctionnelle du phénotype sexuel.

\subsubsection{Croisements entre thermo-néo-mâles $Z W$ et femelles thélygènes WW. -}

Disposant de thermo-néo-mâles $Z W$ et de femelles thélygènes $W W$, il était logique de poursuivre l'analyse génétique par des croisements entre ces animaux à génotype particulier. La descendance théorique issue de ces animaux comporte $50 \%$ de génotypes $Z W$ et $50 \%$ de génotypes WW, ce qui correspond à $100 \%$ de phénotypes femelles si l'élevage est pratiqué à la température ambiante (fig. 1, E).

Les 2 croisements de ce type réalisés à ce jour ont permis d'obtenir pour l'un 57 et pour l'autre 144 animaux après 5 mois d'élevage à la température ambiante. Les laparotomies ont révélé qu'il s'agissait effectivement de $100 \%$ d'individus de phénotype sexuel femelle.

La détection du génotype WW, qui doit se rencontrer théoriquement une fois sur deux dans la descendance issue de tels croisements, devrait être plus commode que dans une population femelle où le génotype $W W$ n'est représenté qu'une fois sur trois. En même temps, il devrait être possible d'avoir des informations plus précises sur la viabilité des animaux ayant une constitution génotypique WW. En outre, l'élevage à température élevée des larves issues de ce type de croisement devrait permettre d'isoler des thermo-néo-mâles WW. 


\section{Discussion.}

L'élevage des jeunes larves d'Amphibiens à une température supérieure à la normale entraîne souvent une perturbation de la sex-ratio au bénéfice du sexe mâle. Connue depuis longtemps, cette observation effectuée à partir d'animaux juvéniles suggérait une inversion du phénotype sexuel femelle liée à l'action de la température, mais aucune preuve d'une telle inversion n'avait été donnée.

Witschi (1929) fut le premier à montrer clairement l'influence de la température sur la différenciation du sexe des gonades. Piquet (1930), Uchida (1937), Yoshikura (1959, 1963) et Hsü, Yü et Liang (1971) n'ont fait que confirmer certaines de ses observations. Witschi élève à $32 \pm 2{ }^{\circ} \mathrm{C}$ des têtards de Rana sy/vatica qui appartient à une race sexuelle différenciée (la gonade mâle se différencie directement en testicule). Le traitement débute après 5 semaines de développement à $20^{\circ} \mathrm{C}$ alors que la différenciation sexuelle de la gonade a déjà commencé. Un premier lot témoin de cet âge comporte 15 mâles et 13 femelles. Après le traitement thermique d'une durée de 15 à 33 jours, il est obtenu 62 mâles, 53 individus possédant des ovaires plus ou moins modifiés, mais aucune femelle avec des ovaires normaux. Un second lot témoin du même âge que les animaux traités comporte 96 mâles et 100 femelles. S'il est clair que les gonades n'ont plus un aspect ovarien typique à la suite du traitement thermique, par contre aucune preuve rigoureuse de l'inversion du phénotype sexuel femelle n'est apportée.

Pour les races sexuelles différenciées auxquelles appartiennent Bufo vulgaris (Piquet, 1930), Rana japonica (Yoshikura, 1959, 1963) et Rana catesbeiana (Hsü, Yü et Liang, 1971) l'action d'une température supérieure à la normale paraît affecter les femelles puisque, en référence à la sex-ratio, les gonades intersexuées ne peuvent résulter que de l'inversion partielle des gonades femelles.

Le seul Urodèle étudié auparavant́ quant à l'action de la température d'élevage, Hynobius retardatus, appartient à une espèce semi-différenciée (Uchida, 1937) ; les gonades mâles passent par un stade ovarien transitoire et ne sont pas encore totalement différenciées en testicules à l'époque de la métamorphose. Des larves d'âges différents ont subi des traitements à $30^{\circ} \mathrm{C}$ d'une durée variant entre 8 et 71 jours. Cet Urodèle s'élevant difficilement au laboratoire, les larves ont été sacrifiées avant la métamorphose. Le principal résultat rapporté est une masculinisation plus ou moins profonde des gonades en rapport avec la durée du traitement. Mais l'interprétation des images histologiques doit tenir compte du caractère semi-différencié de l'espèce en cause.

Ainsi, l'originalité du présent travail est d'apporter pour la première fois chez les Vertébrés Tétrapodes la démonstration génétique de l'inversion fonctionnelle d'un phénotype sexuel sous l'action d'un traitement thermique. L'expérience initiale (Houillon et Dournon, 1978) était déjà par elle-même très démonstrative puisque des animaux à génotype sexuel femelle connu, élevés à $30^{\circ} \mathrm{C}$ dès le stade de l'éclosion, manifestaient tous les caractères externes de la maturité sexuelle mâle à l'âge de 6 mois. Les expériences ultérieures (Dournon et Houillon, 1983) ont apporté trois preuves supplémentaires d'ordre génétique reposant sur l'existence de ces thermo-néo-mâles fonctionnels. 
La détection des génotypes sexuels est basée sur l'analyse statistique de la descendance. Malgré ses contraintes, cette méthode est la plus rigoureuse dans le cas de Pleurodeles waltlii. La remarquable conformité des sex-ratio observées par rapport aux prévisions théoriques la rend particulièrement appropriée pour la démonstration de l'inversion fonctionnelle d'un phénotype sexuel. L'analyse enzymatique basée sur la peptidase-1 est une méthode rapide, applicable très tôt au cours du développement. Cependant, elle nécessite de connaître le phénotype enzymatique des ascendants afin d'éviter les croisements à partir desquels les descendants à génotype $Z Z$ et $Z W$ pourraient avoir le même zymogramme. L'analyse statistique, associée à l'analyse enzymatique, vient de conduire à la sélection de Pleurodèles dont la descendance peut être identifiée par la seule analyse enzymatique. Cette double étude a permis la détection des thermo-néo-mâles WW. L'ensemble de ces résultats confirme l'indépendance de l'expression de la peptidase-1 par rapport à l'environnement hormonal ainsi que l'a démontrée Ferrier et al. (1983) notamment à partir d'un thermo-néo-mâle ZW obtenu selon notre propre technique d'inversion du phénotype sexuel.

L'inversion du phénotype sexuel mâle sous l'action du benzoate d'œstradiol avait été obtenue par Gallien (1950) et l'inversion du phénotype sexuel femelle par Collenot (1973) grâce aux greffes embryonnaires. Signalons également l'inversion du phénotype sexuel femelle dans le cas particulier de la greffe embryonnaire en chimère (Houillon, 1977 ; Houillon et Dournon, 1980). Comme pour le présent travail, les preuves génétiques de ces inversions reposent sur l'analyse de la descendance des animaux à phénotype sexuel inversé et permettent d'affirmer que malgré l'absence de chromosomes sexuels morphologiquement discernables, il existe chez Pleurodeles waltlii un déterminisme génétique du sexe basé sur l'homogamétie mâle (ZZ) et l'hétérogamétie femelle (ZW).

L'action de la température sur la différenciation du sexe est davantage étudiée chez les Reptiles. Charnier (1966) suggéra que la sex-ratio pourrait dépendre de la température d'incubation des œufs chez un lézard Agama agama. Chez deux espèces de tortue Emys orbicularis et Testudo graeca, Pieau $(1971,1972)$ fut le premier à observer que l'incubation des embryons à $30-32{ }^{\circ} \mathrm{C}$ conduisait à $100 \%$ de femelles alors que l'incubation à $25-27{ }^{\circ} \mathrm{C}$ conduisait à $100 \%$ de mâles. Ces travaux précurseurs viennent d'être étendus avec des résultats du même ordre à plusieurs autres espèces de Chéloniens, à un lézard (revue de Bull, 1980) et plus récemment à un alligator (Ferguson et Joanen, 1982). Cependant la thermosensibilité de la différenciation sexuelle ne s'observe pas chez tous les Reptiles. Certains n'ont manifesté aucune modification de la sex-ratio à la suite de traitements thermiques, du moins dans les conditions qui leur ont été appliquées. C'est le cas de diverses espèces aussi bien de lézards (Raynaud et Pieau, 1972 ; Muth et Bull, 1981) que de serpents et de quelques tortues (Bull, 1980). L'inversion d'un phénotype sexuel sous l'action de la température chez les Reptiles a été déduite de l'obtention, sans mortalité différentielle, de $100 \%$ d'individus du même sexe selon le traitement thermique, mais aucune preuve génétique n'a encore été apportée quant à l'aspect fonctionnel et stable de cette inversion.

Chez les Poissons, deux espèces dont l'une hermaphrodite dans les conditions naturelles Rivulus marmoratus (Harrington, 1967) et l'autre gonochorique 
Menidia menidia (Conover et Kynard, 1981) sont actuellement connues pour la sensibilité à la température de la différenciation de leurs gonades.

L'inversion obtenue par les greffes embryonnaires nécessite une technologie assez lourde qui a le double inconvénient de n'être applicable qu'à un nombre réduit d'animaux et seulement chez les Amphibiens. Par contre, l'utilisation de températures d'élevage différentes des conditions habituelles est une technologie très simple aussi bien pour les Amphibiens que pour les Reptiles. Applicable à un grand nombre d'animaux en même temps, cette technologie permet une approche biochimique des facteurs impliqués dans la différenciation sexuelle. L'avantage du Pleurodèle par rapport aux tortues pour une telle étude est que la maturité sexuelle est atteinte plus rapidement et que l'on dispose de femelles reproductrices aussi bien thélygènes $W W$ qu'arrhénogènes $Z Z$. Croisées avec des mâles standard $Z Z$ les premières donnent une descendance à génotype $Z W$ et les secondes une descendance à génotype $Z Z$. De la sorte, les analyses biochimiques peuvent s'effectuer à partir d'animaux dont le génotype est connu en toute certitude quel que soit le stade de développement et quelles que soient les conditions de température.

L'action de la température sur la différenciation du sexe est analysée dans le présent travail en fonction de deux conditions fort différentes: les animaux témoins sont élevés à la température ambiante qui varie entre 18 et $22^{\circ} \mathrm{C}$ pour laquelle la sex-ratio est normale, alors que les animaux expérimentaux sont élevés à la température de $30^{\circ} \mathrm{C}$, très supérieure à la normale, toujours constante et pour laquelle on constate un net excès de mâles. En plus de la température ellemême, on pourrait concevoir une action due à ses fluctuations chez les témoins et à sa constance chez les animaux en expérience. Il est probable qu'une température constante de $20^{\circ} \mathrm{C}$ conduise à une sex-ratio normale, encore faudrait-il le vérifier. L'étude des températures constantes, supérieures ou inférieures de quelques degrés par rapport à $20^{\circ} \mathrm{C}$ n'a jamais été entreprise d'une manière systématique. Cette étude serait d'ailleurs délicate puisqu'il a été constaté une thermosensibilité différente selon les pontes pour la température de $30^{\circ} \mathrm{C}$. II n'en demeure pas moins que l'action des fluctuations de température qui doivent se compenser si la sex-ratio est normale, est encore inconnue.

Ce dernier point amène à considérer l'identité des mécanismes de la différenciation sexuelle chez les Vertébrés poïkilothermes et homéothermes. Pour les premiers, la différenciation normale du sexe, c'est-à-dire conforme au sexe génétique, s'effectue entre certaines limites de température. En deçà ou au-delà de ces limites, la différenciation est perturbée et même modifiée. Pour les homéothermes, la différenciation sexuelle s'effectue à des températures constantes mais d'une élévation telle qu'appliquées à un quelconque poïkilotherme, elles entraîneraient la mort des individus. Les mécanismes en cause relèvent sans conteste de processus métaboliques que les homéothermes ont acquis au cours de l'évolution en se libérant des variations de température et en s'adaptant à des conditions de température élevée. Peut-être pourrait-on invoquer ici la participation de phénomènes de cette nature dans la disparition de certains grands Reptiles poïkilothermes ovipares. Ne disposant pas encore des mécanismes de régulation des homéothermes, ils ont fini par disparaître lorsque des conditions de température particu- 
lières sont intervenues favorisant la diftérenciation préférentielle d'un sexe (Pieau, 1982 ; Ferguson et Joanen, 1982). Pieau (1974, 1982) et Bull et Vogt (1979) ont montré l'importance de la température du sol pendant la période de la différenciation sexuelle des glandes génitales chez les tortues. Dans les conditions naturelles, lorsque la température oscille quotidiennement de part et d'autre de la température critique $\left(28-29^{\circ} \mathrm{C}\right)$ la sex-ratio est équilibrée, on trouve des mâles, des femelles et quelquefois des intersexués. Mais au-dessus ou au-dessous de ce seuil, on constate un net excès de femelles ou bien un net excès de mâles.

Chez les Amphibiens étudiés, la différenciation des deux sexes s'effectue à des températures plus basses et dans une gamme de température plus large que chez les tortues. Les homéothermes différencient les deux sexes à une température constante élevée. Chez les Reptiles actuels la différenciation des deux sexes s'effectue à des températures plus élevées et dans une gamme plus étroite que chez les Amphibiens. On peut se demander s'il ne s'agit pas là d'un vestige de leur tentative d'approche vers l'homéothermie.

Au même titre que les hormones sexuelles et les greffes embryonnaires, la température d'élevage anormalement élevée est un facteur épigénétique qui intervient pour modifier l'expression du programme génétique de la détermination du sexe mis en place au moment de la fécondation. La gonade d'un Vertébré présente une bipotentialité anatomique aux stades embryonnaires ; ce n'est que relativement tard par rapport à d'autres phénomènes de différenciation qu'il se fera un choix entre la différenciation testiculaire et la différenciation ovarienne. Or chez le Pleurodèle, on peut modifier l'expression du programme génétique par la température. L'intérêt de ce modèle est qu'il devrait permettre d'analyser les mécanismes cellulaires et moléculaires encore inconnus qui interviennent au tout début de la différenciation sexuelle des Vertébrés.

Remerciements. - Travail effectué avec l'aide financière de la DGRST, contrat $n^{\circ}$ 79.7.1221, puis du CNRS, ATP «Biologie du Développement et de la Reproduction ».

\section{Références}

BULL J. J., 1980. Sex determination in reptiles. Quart. Rev. Biol., 55, 3-21.

BULL J. J., VOGT R. C., 1979. Temperature-dependent sex determination in turtles. Sciences, 206, 1186-1188.

CAYROL C., JAYLET A., FERRIER V., GASSER F., 1983. A genetic study of various enzyme polymorphisms in Pleurodeles walt/ii (Urodele, Amphibian). Ill-The relationship between sexlinked peptidase-1 expression and gene-dose effects. Biochem. Genet, 21, 551-559.

CHARNIER M., 1966. Action de la température sur la sex-ratio chez l'embryon d'Agama agama (Agamidae, Lacertilien), C. R. Soc. Biol., 160, 620-622.

COLLENOT A., 1973. Obtention, par la méthode des greffes de gonades embryonnaires, d'une femelle à descendance unisexuée femelle, chez le triton Pleurodeles walt/ii Michah. Experientia, 29, 885-887.

CONOVER D. O., KYNARD B. E., 1981. Environmental sex determination : interaction of temperature and genotype in a fish. Science, 213, 577-579. 
DOURNON C., HOUILLON Ch., 1983. Déterminisme génétique du sexe : démonstration à partir d'animaux à phénotype sexuel inversé sous l'action de la température chez l'Amphibien Urodèle, Pleurodeles waltlii Michah. C. R. Acad. Sci. Paris, Sér. III, 296, 779-782.

FERGUSON M. W., JOANEN T., 1982. Temperature of egg incubation determines sex in Alligator mississippiensi. Nature, 296, 850-853.

FERRIER V., JAYLET A., CAYROL C., GASSER F., BUISAN J. J., $1980 . \quad$ Etude électrophorétique des peptidases érythrocytaires chez Pleurodeles waltlii (Amphibien Urodèle) : mise en évidence d'une liaison avec le sexe. C. R. Acad. Sci. Paris, Sér. D, 290, 571-574.

FERRIER V., GASSER F., JAYLET A., CAYROL C., 1983. A genetic study of various enzyme polymorphisms in Pleurode/es waltlii (Urodele Amphibian). II-Peptidases : demonstration of sex linkage. Biochem. Genet., 21, 535-549.

GALLIEN L., 1950. Inversion du sexe (féminisation) chez l'Urodèle Pleurodeles waltii Michah. traité par le benzoate d'œestradiol. C. R. Acad. Sci. Paris, 231, 919-920.

GALLIEN L., 1951. Sur la descendance unisexuée d'une femelle de Pleurodeles waltiii Michah. ayant subi pendant sa phase larvaire l'action gynogène du benzoate d'cestradiol. $C$. R. Acad. Sci. Paris, 233, 828-830.

GALLIEN L., 1952. Elevage et comportement du Pleurodèle au laboratoire. Bull. Soc. Zool. Fr., 77, 456-461.

GALLIEN L., 1954. Inversion expérimentale du sexe sous l'action des hormones sexuelles chez le triton Pleurodeles waltlii Michah. Analyse des conséquences génétiques. Bull. Biol. Fr. Belg., 88, 1-51.

GALLIEN L., DUROCHER M., 1957. Table chronologique du développement chez Pleurodeles waltlii Michah. Bull. Biol. Fr. Belg., 91, 97-114.

HARRINGTON R. W., 1967. Environmentally controlled induction of primary male gonochorists from eggs of the self-fertilizing hermaphroditic fish, Rivulus marmoratus Poey. Biol. Bull., 132, 174-199.

HERTWIG O., 1898. Über den Einfluss der Temperatur auf die Entwicklung von Rana fusca und Rana esculenta. Arch. mikr. Anat. Entw., 51, 319-381.

HOUILLON Ch., 1977. Tractus uro-génital des chimères chez l'Amphibien Urodèle Triturus alpestris Laur. J. Embryol. exp. Morphol., 42, 15-28.

HOUILLON Ch., DOURNON C., 1978. Inversion du phénotype sexuel femelle sous l'action d'une température élevée chez l'Amphibien Urodèle, Pleurodeles waltiii Michah. C. R. Acad. Paris, Sér. $D, 286,1475-1478$.

HOUILLON Ch., DOURNON C., 1980. Preuves génétiques et immunologiques de l'inversion du sexe dans les chimères hétérosexuées chez les Urodèles. Arch. Anat. Micr. Morph. exp., 69, 320-321.

HSÜ C. Y., YÜ N. W., LIANG H. M., 1971. Induction of sex reversal in female tadpoles of Rana catesbeiana by temperature treatment. Endocrinol. japon., 18, 243-251.

HUMPHREY R. R., 1928. Sex differentiation in gonads developed from transplants of the intermediate mesoderm of Amblystoma. Biol. Bull., 55, 317-339.

HUMPHREY R. R., 1931. Studies on sex reversal in Amblystoma. III-Transformation of the ovary $A$. tigrinum into a functional testis through the influence of a testis resident in the same animal. J. exp. Zool., 58, 333-366.

HUMPHREY R. R., 1945. Sex determination in ambystomid salamanders : a study of the progeny of females experimentally converted into male. Amer. J. Anat., 76, 33-66.

MUTH A., BULL J. J., 1981. Sex determination in desert Iguanas does incubation temperature make a difference? Copeia, 4, 869-870.

PIEAU C., 1971. Sur la proportion sexuelle chez les embryons de deux Chéloniens (Testudo graeca L. et Emys orbicularis L.) issus d'œufs incubés artificiellement. C. R. Acad. Sci. Paris, Sér. $D, 272,3071-3074$.

PIEAU C., 1972. Effets de la température sur le développement des glandes génitales chez les embryons de deux Chéloniens, Emys orbicularis L. et Testudo graeca L. C. R. Acad. Sci. Paris, Sér. D, 274, 719-722.

PIEAU C., 1974. Sur la différenciation sexuelle chez les embryons d'Emys orbicularis L. (Chéloniens) issus d'œufs incubés dans le sol au cours de l'été 1973. Bull. Soc. Zool. Fr., 99, 363-376. 
PIEAU C., 1982. Modalities of the action of temperature on sexual differentiation in fielddeveloping embryos of the european pond turtle Emys Orbicularis (Emydidae). J, exp. Zool., 220, 353-360.

PIQUET J., 1930. Détermination du sexe chez les Batraciens en fonction de la température. Rev. suisse Zool., 37, 173-281.

RAYNAUD A., PIEAU C., 1972. Effets de diverses températures d'incubation sur le développement somatique et sexuel des embryons de Lézard vert (Lacerta viridis Laur.). C. R. Acad. Sci. Paris, Sér. D, 275, 2259-2262.

UCHIDA T., 1937. Studies of the sexuality of Amphibia. III-Sex transformation in Hynobius retardatus by high temperature. J. Fac. Sci., Hokkaïdo Imp. Univ., ser. 6 (Zool.), 6, 59-70.

WITSCHI E., 1914. Experimentelle Untersuchungen über die Entwicklungsgeschichte der keimdrüsen von Rana temporaria. Arch. mikr. Anat. Entw., 85, 9-113.

WITSCHI E., 1929. Studies on sex differentiation and sex determination in Amphibians. II-Sex reversal in female tadpoles of Rana sy/vatica following the application of high temperature. $J$. exp. Zool., 52, 267-291.

YOSHIKURA M., 1959. The action of the pituitary in sex differentiation and sex reversal in Amphibians. II-Effects of high temperature on the gonads of hypophysectomized frog larvae. Kumamoto J. Sci., ser. B, sec. 2, 4, 69-101.

YOSHIKURA M., 1963. Influence of high temperature on the development of gonads of thioureatreated frog tadpoles. Kumamoto J. Sci., ser. B, sec. 2, 6, 79-101. 